Ann. Zootech., I969, 18 (4), 437-443.

\title{
UTILISATION DIGESTIVE RÉELLE ET RÉTENTION DU CALCIUM PAR LE LAPIN EN CROISSANCE RECEVANT UN RÉGIME RICHE EN CALCIUM ET EN PHOSPHORE
}

\author{
P. BESANÇON et F. LEBAS \\ avec $^{-}$la collaboration technique de Colette KaTz \\ Station centrale de Nutrition, \\ Station de Recherches sur l'Élevage des Porcs, \\ Centre national de Recherches zootechniques, 78-Jouy-en-Josas \\ Institut national de la Recherche agronomique
}

\section{SOMMAIRE}

L'utilisation digestive réclle et la rétention du calcium ont été estimées sur des lapins Fauve de Bourgogne, de $\mathrm{I}_{3}$ semaines, recevant un régime riche en calcium et en phosphore, contenant I7 $\mathrm{g}$ de calcium et I $\mathrm{I} g$ de phosphore par $\mathrm{kg}$ de matière sèche. L'excrétion fécale de calcium endogène a été mesurée par une méthode de dilution isotopique après une injection intraveineuse de calcium radioactif. Le niveau d'ingestion calcique est de 3,2 g par jour et dépasse très largement le besoin optimal de l'animal. Le calcium ingéré semble bien utilisé au niveau digestif, avec un CUD réel de $5 \mathrm{I}, 8 \mathrm{p}$. Ioo, mais le calcium absorbé cst mal fixé (coefficient de rétention de 27,6 p. I00 de l'ingéré). Cette faible rétention est due à une forte élimination du calcium endogène par les fèces $(0,27 \mathrm{~g}$ par jour) et par les urines $(0,47 \mathrm{~g}$ par jour). Des apports phosphocalciques moindres seraient probablement mieux utilisés.

\section{INTRODUC'TION}

Les aliments à usages multiples couramment utilisés pour l'élevage du Lapin, et qui permettent de couvrir aussi bien les besoins de croissance que ceux de lactation, sont en général très riches en calcium et en phosphore. On peut se demander dans quelle mesure cela est justifié pour l'animal en croissance, dont les besoins phosphocalciques sont plus réduits. On sait en effet que 1'utilisation du calcium et du phosphore est moins bonne si ces éléments minéraux se trouvent en excès dans l'alimentation. 
Nous nous sommes donc proposés de mesurer les bilans calciques et l'utilisation digestive réelle du calcium sur des lapins en croissance recevant un régime dont les teneurs en phosphore et en calcium sont très élevées et, en l'absence d'un lot témoin, de comparer les résultats à ceux obtenus par ailleurs sur le Lapin ou d'autres espèces, avec des apports phosphocalciques moindres ou équivalents.

\section{MATÉRIEL ET MÉTHODES}

Dix lapins Fauves de Bourgogne en croissance ( 5 mâles et 5 femelles), âgés de I 3 semaines et pesant $2,5 \mathrm{~kg}$ en moyenne, ont été mis en cages à métabolisme. Ils recevaient ad libitum de l'eau et un aliment sous forme de granulés dont la composition était la suivante : orge : I $5 \mathrm{p}$. Ioo ; avoine: 20 p. Ioo; son de blé : Io p. Ioo; tourteau de soja cuit: I 5 p. Ioo; tourteau de tournesol: 5 p. Ioo ; farine de luzerne déshydratée : 30 p. I00 ; levure : 1 p. Ioo ; complément minéral vitaminique : 4 p. Ioo. L'aliment contenait $\mathrm{r} 7 \mathrm{~g}$ de calcium et I $\mathrm{g}$ de phosphore par $\mathrm{kg}$ de matière sèche. Ces niveaux sont très élevés par rapport aux besoins minima de l'animal ; en effet, MATHIEU et SMrth (196I) et ChAPIN et Smith (I967 a) ont montré sur des lapins Néo-zélandais blancs que 2,2 p. I ooo de $\mathrm{P}$ et Ca permettent de satisfaire les besoins minima et d'assurer l€s meilleures performances zootechniques. Le besoin optimal se situerait entre 3,5 et 4,0 p. I ooo pour le calcium, teneur qui entraîne une minéralisation osseuse supérieure.

Après une période d'accoutumance d'une semaine, urines et fèces ont été recueillies séparément et en totalité durant 7 jours. Dès le début de la période de mesure des bilans calciques, une injection de ${ }^{45} \mathrm{Ca}$ (environ $90 \mu \mathrm{Ci}$ sous forme de $\mathrm{CaCl}_{2}$ ) a été réalisée par la veine marginale de l'oreille et du sang a été prélevé périodiquement dans l'oreille non contaminée par l'injection de radiocalcium. Nous avons ainsi suivi la décroissance de l'activité spécifique du calcium plasmatique durant 5 à 7 jours ; le rapport des activités spécifiques du calcium plasmatique et du calcium fécal après 5 jours permet d'estimer la fraction de Ca endogène dans l'excrétion fécale par la méthode de dilution isotopique proposée par LofGREEN et KLEIBER (1954) pour le phosphore.

Le calcium a été dosé par photométrie d'émission de flamme sur les échantillons minéralisés au four à $55^{\circ} \mathrm{C}$. La radioactivité a été mesurée au moyen d'un compteur Geiger-Müller à fenêtre mince et à flux gazeux d'hélium.

\section{RÉSULTATS}

Le gain de poids moyen des animaux a été de $20 \mathrm{~g}$ par jour, ce qui représente une croissance satisfaisante pour des lapins mis en cage à métabolisme. Pratiquement, les animaux se trouvent à la fin de la phase de croissance rapide.

Les valeurs des bilans calciques sont groupées dans le tableau I. L'utilisation digestive apparente du calcium, calculée à partir de la différence entre l'ingéré et l'excrété fécal total, représente $42, \mathrm{I}$ p. roo de l'ingéré et semble donc élevée, mais ne donne pas une idée précise de ce qui est effectivement absorbé. I a connaissance des pertes fécales de calcium endogène permet d'estimer le coefficient d'utilisation digestive réelle (CUD réel) du calcium du régime. Ces paramètres n'ont pas pu être mesurés pour les lapins 6 et 9 sur lesquels il n'a pas été possible de prélever du sang suffisamment longtemps après l'injection de ${ }^{45} \mathrm{Ca}$. En moyenne, I4,o p. Ioo des pertes fécales de calcium sont d'origine endogène; ainsi, l'excrétion fécale de calcium exogène représente en valeur réelle 48,2 p. Ioo de l'ingéré. C'est pourquoi on passe d'un CUD apparent de 42,I p. Ioo à un CUD réel de 5r,8 p. Ioo.

En revanche, la rétention calcique n'est plus que de $27,6 \mathrm{p}$. Ioo de l'ingéré. L'écart très grand qui apparaît entre le CUD réel et le coefficient de rétention est dû à l'importance de l'élimination de calcium endogène par les fèces $(0,27 \mathrm{~g}$ par jour) et par les urines (o,47 g par jour). 


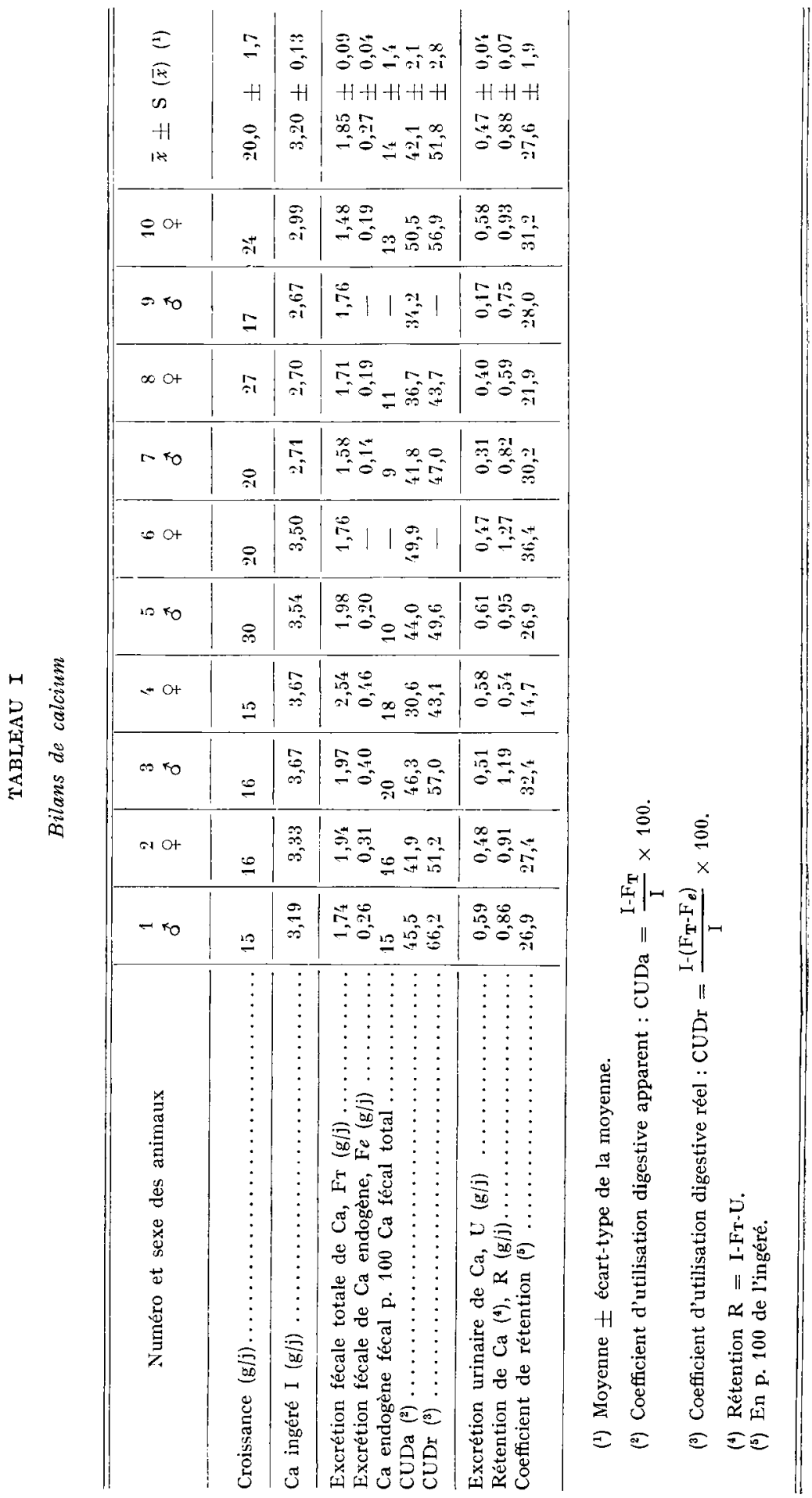


Le tableau 2 montre qu'il n'y a de différence significative entre mâles et femelles sur aucun des paramètres du métabolisme calcique.

TABLEAU 2

Comparaison entre mâles et femelles

(Moyenne \pm écart type de la moyenne)

\begin{tabular}{|c|c|c|}
\hline & Mâles & Femelles \\
\hline Croissance $(g / j)$. & $19,6+2,7$ & $20,4+2,2$ \\
\hline Ca ingéré $(\mathrm{g} / \mathrm{j}) \ldots \ldots \ldots \ldots \ldots \ldots$ & $3,16 \pm 0,21$ & $3,24 \pm 0,18$ \\
\hline Excrétion fécale totale de $\mathrm{Ca}(\mathrm{g} / \mathrm{j})$ & $1,81 \pm 0,08$ & $1,89 \pm 0,18$ \\
\hline Excrétion fécale de Ca endogène $(\mathrm{g} / \mathrm{j})$ & $0,25 \pm 0,06$ & $0,29 \pm 0,06$ \\
\hline CUD apparent (p. $100 \mathrm{Ca}$ ingéré) .... & $42,1 \pm 2,2$ & $41,9 \div 3,8$ \\
\hline CUD réel (p. $100 \mathrm{Ca}$ ingćrć) $\ldots .$. & $55,0 \pm 4,3$ & $48,7 \pm 3,3$ \\
\hline Excrétion urinaire de $\mathrm{Ca}(\mathrm{g} / \mathrm{j}) \ldots$ & $0,44 \pm 0,09$ & $0,50 \pm 0,03$ \\
\hline Rétention de $\mathrm{Ca}(\mathrm{g} / \mathrm{j}) \quad \ldots \ldots \ldots \ldots \ldots \ldots \ldots \ldots \ldots \ldots \ldots \ldots \ldots$ & $0,91 \pm 0,08$ & $0,85 \pm 0,13$ \\
\hline Coefficient de rétention de $\mathrm{Ca}$ (p. $100 \mathrm{Ca}$ ingéré) & $28,9 \pm 1,1$ & $26,3 \pm 3,7$ \\
\hline
\end{tabular}

DISCUSSION

\section{Utilisation digestive du calcium}

Malgré l'apport élevé de calcium dans notre régime, l'utilisation digestive apparente de $\mathrm{Ca}$ est satisfaisante puisqu'elle est en moyenne de 42 p. Ioo de 1'ingéré. Toutefois, STERNKOPF ( $\mathrm{rg}_{63}$ ) trouve des valeurs du coefficient d'utilisation digestive apparente (CUD apparent) plus élevées sur des lapins Chinchilla de $2,7 \mathrm{~kg}$, recevant un régime à base de légumineuses, luzerne ou trèfle rouge. Cette meilleure digestibilité peut s'expliquer par le fait que l'ingestion calcique est plus faible que dans nos essais, malgré une teneur en calcium très élevée dans 1'aliment. Suzuki (I962, I963a) a trouvé a fortiori des valeurs du CUD apparent beaucoup plus importantes sur des lapins de race Japonaise blanche, de I,I à I, $2 \mathrm{~kg}$, ingérant des quantités de calcium six à huit fois moindres. Il faut remarquer aussi que les différentes sources de calcium ne sont pas toutes utilisées de la même façon (SUZUkI, I962) et que le besoin minéral et 1'utilisation digestive du calcium et du phosphore sont directement liés à la composition et à la nature du régime, comme l'ont montré Wooley et MICKELSEN (I954)

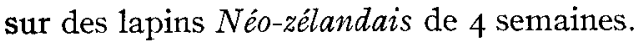

Cependant, d'après Chapin et Smith $(\operatorname{Ig} 67 b)$, le Lapin en croissance tolère fort bien des régimes très riches en calcium, allant jusqu'à $40 \mathrm{p}$. I ooo de $\mathrm{Ca}$; le niveau de phosphore peut aussi être augmenté dans de fortes proportions pourvu que 1'apport calcique soit accru proportionnellement (Chapin et SMith, I967 c). Cela peut expliquer l'utilisation digestive satisfaisante du calcium de notre régime très riche mais équilibré en $\mathrm{Ca}$ et $\mathrm{P}$.

Enfin, le niveau élevé de l'absorption digestive du calcium est probablement une des conséquences du phénomène de coprophagie, que nous n'avons pas cherché à empêcher. 


\section{Excrétion fécale de calcium endogène}

Si les pertes fécales de calcium endogène ne semblent pas très élevées lorsqu'elles sont exprimées en pourcentage de l'excrétion totale de calcium par les fèces, elles sont, en revanche, très fortes en valeur absolue. Cette élimination apparaît encore plus importante si on la rapporte au poids de l'animal : Io7 $\mathrm{mg}$ de calcium endogène fécal par $\mathrm{kg}$ de poids vif et par jour, soit une valeur quatre à dix fois supérieure à celle que l'on rencontre chez les autres mammifères domestiques, même lorsqu'ils reçoivent des apports élevés de calcium (HANSARd et PlumleE, I954 $a$; HANSARD et Crowder, I957). Plusieurs hypothèses pourraient expliquer ces résultats :

a) L'apport phosphocalcique très élevé entraîne une moins bonne rétention et une sorte de gaspillage du calcium absorbé : celui-ci se trouve alors excrété par voie fécale ou urinaire. Cette hypothèse est aussi confirmée par les résultats de SuzukI $($ I963 b) qui, sur des lapins Japonais blancs recevant un régime pratiquement dépourvu de calcium (ingéré calcique de 4 à $40 \mathrm{mg}$ par jour), a mesuré une excrétion fécale de calcium endogène de 25 à $35 \mathrm{mg}$ par jour, ce qui représente dans ce cas Io,6 $\mathrm{mg}$ de $\mathrm{Ca}$ par $\mathrm{kg}$ de poids vif, soit dix fois moins que dans nos essais. Cependant, Suzuki fait remarquer que la méthode employée, qui consiste à rendre nul l'apport calcique, conduit à des valeurs de l'excrétion fécale d'origine endogène plus faibles que celles que l'on obtient à l'aide de méthodes radioisotopiques. Il est sûr, d'autre part, que de telles conditions nutritionnelles ne peuvent pas être considérées comme normales.

b) Il est par ailleurs probable que les pertes fécales de calcium endogène augmentent avec l'âge et qu'elles seraient plus faibles pour des animaux plus jeunes ayant une vitesse de croissance plus élevée.

c) Enfin, cette importante excrétion fécale de calcium endogène pourrait être aussi considérée comme une caractéristique de l'espèce. D'après les résultats de Suzukr ( $\mathrm{I}^{6} 63 \mathrm{~b}$ ), cela ne semble pas vraisemblable. Néanmoins, il ne nous est pas permis d'abandonner totalement cette hypothèse, car de tels écarts de l'excrétion fécale endogène ne sont pas fréquents chez les autres mammifères recevant des régimes riches en calcium. Il n'est donc pas exclu qu'il y ait chez le Lapin un type particulier de régulation au niveau digestif, la coprophagie n'y étant peut-être pas étrangère.

\section{Rétention calcique et rétention de la radioactivité injectée}

La forte élimination urinaire de calcium, qui a pour conséquence de rendre assez faible la rétention, confirme l'hypothèse faite plus haut d'une mauvaise fixation du calcium absorbé. Il est intéressant de remarquer aussi que la rétention du phosphore n'est que de 29 p. roo (données non publiées). Mais, l'élévation de la calciurie sous l'effet d'apports calciques excessifs, rare chez les mammifères domestiques, est probablement aussi une caractéristique du Lapin.

De même, la rétention de la dose de radioactivité administrée n'est que de 52 p. Ioo au bout de 6 jours, ce qui semble très faible par rapport aux valeurs troutvées sur d'autres espèces (HANSARD et al., I954 $b$; BESANÇON et al., I966). Nous avons mesuré que $30 \mathrm{p}$. Ioo de la dose est excrétée par la voie urinaire et I 8 p. Ioo par la voie fécale. Les pertes fécales sont comparables aux valeurs obtenues sur des lapins 
adultes par Thomas et al. (I952), mais les auteurs ne précisent ni l'âge, ni le poids ni la race de leurs animaux; ils montrent cependant que chez le jeune l'élimination fécale est moins rapide. Les valeurs de l'excrétion urinaire de la dose que nous avons mesurées au bout de 6 jours sont intermédiaires entre les résultats trouvés sur le jeune et l'adulte par ces mêmes auteurs.

Il serait intéressant de savoir comment le calciun absorbé et retenu est utilisé au niveau de l'os et quelles sont les caractéristiques dynamiques du métabolisme minéral osseux : vitesses d'incorporation et de mobilisation du calcium, intensité du renouvellement minéral osseux. 'lHomas et al. (I952), en comparant les courbes de décroissance de l'activité spécifique du calcium sanguin après une injection de ${ }^{45} \mathrm{C}$ a montrent que, chez le jeune, l'os incorpore le calcium deux fois plus vite que chez l'adulte, mettant ainsi en évidence des différences relatives d'activité métabolique de l'os, mais ils ne donnent pas les valeurs absolues des vitesses d'entrée et de sortie du calcium osseux. La connaissance de ces paramètres osseux permettrait de mieux interpréter les résultats de bilans calciques que nous avons obtenus.

En conclusion, des apports calciques dépassant très largement le besoin optimal semblent bien utilisés au niveau digestif par le Lapin, puisque l'utilisation digestive réelle du calcium est de 52 p. Ioo de l'ingéré. İn revanche, le calcium absorbé est mal retenu : le coefficient de rétention n'est que de 28 p. Ioo de l'ingéré, cette mauvaise rétention étant due à la forte élimination de calcium endogène par les urines et les fèces.

Le Lapin, soumis à de hauts niveaux d'ingestion calcique, présente clonc la particularité de ne pas réduire sa capacité d'absorption intestinale, comme le font généralement les autres espèces et, en conséquence, il doit accroître simultanément ses excrétions fécales et urinaires de calcium endogène. Une autre différence tient au fait que les mammifères domestiques herbivores, recevant des excès de calcium, n'excrètent pas plus de calcium par les urines, mais augmentent leur excrétion fécale de calcium endogène, dans des linites toutefois moindres que celles que nous observons chez le Lapin.

Par ailleurs, 1'accroissement des apports calciques entraîne une augmentation de la minéralisation osseuse. Il serait nécessaire de déterminer le seuil au-dessus duquel il y a gaspillage de calcium absorbé, celui-ci ne pouvant plus être retenu ; ce niveau serait encore probablement supérieur à un niveau optimal permettant une ossification répondant à des critères dynamiques satisfaisants. Ėn l'absence de telles données, il est encore prématuré de formuler des recommandations pratiques précises sur le besoin calcique dı Lapin. Toutefois, il est sîr que des apports phosphocalciques tels que ceux que nous avons offerts à nos animaux sont très élevés par rapport aux besoins de l'animal en croissance et que des niveaux de $\mathrm{P}$ et de Ca moindres en assureraient une meilleure utilisation. 


\title{
SUMMARY
}

\author{
TRUE DIGESTIVE UTIIIZATION AND CAICIUM RETENTION IN THE GROWING \\ RABBIT RECEIVING A CAICIUM AND I'HOSPHORUS RICH DIET
}

True digestive utilization and calcium retention have been estimated in I 3 -week old liauve de Bourgogne rabbits receiving a calcium and phosphorus rich dict containing I $7 \mathrm{~g}$ Ca and I I g P per $\mathrm{kg}$ of dry weight. Fecal excretion of endogenous calcium has been measured by an isotopic dilution method following an intravenous injection of $90 \mu \mathrm{Ci}$ of +5 -calcium. The high level of calcium intake of $3.20 \pm 0.13 \mathrm{~g}$ per day is much greater than the optimum need of the animal. Ingested calcium seems to be well absorbed, since the true digestive utilization coefficient is 5 I. $8 \pm 2.8 \mathrm{p}$. 100 , but absorbed calcium is not well retained, the retention coefficient being only $27.6 \pm 1.9$ p. 100 of the intake. This low retention is due to a large elimination of en clogenous calcium in the feces, 0.27 上 0.04 \& per day ( 4 p. 1 oo of total fecal calcium loss), and in the urine, $0.47 \pm 0.04 \mathrm{~g}$ per day. Fecal calcium excretion represents $107 \mathrm{mg}$ per $\mathrm{kg}$ of live weight and per day. This is very much higher than values obtained in other domestic animals. Thus, the rabbit, when given a high ingestion level of calcium, presents the peculiarity of not reducing its intestinal absorption and should, consequently, simultaneously increase its fecal and urinary endogenous calcium excretion. A lower phosphocalcic intake would probably be better utilized.

\section{RÉFÉRENCES BIBL,IOGRAPHIQUES}

Besançon P., Gulgues L., Rérat A., ig66. Fehanges et vitesse d'incorporation du calcium dans le squelette du porc en croissance. C. R. Acad. Sci. Paris, 263, I I34-I I37.

Chapin R. E., Smith S. E., I967a. Calcium requirement of growing rabbits. I. Anim. Sci, 26,67-7I.

ChapiN R. E., Sмith S. L., $1967 b$. The calcium tolerance of growing and reproducing rabbits. Cornell Vet., 57, 480-49I.

Chapin R. E., Smitu S. E., I967c. High phosphorus diets fed to growing rabbits. Cornell Ict., 57 492-500.

Hansard S. L., PLumleE M. P., I954a. Effects of dietary calcium and phosphorus levels upon the physiological behaviour of calcium and phosphorus in the rat. J. Nutrition, 54, I7-3I.

Hansard S. L., Comar C. L., Plumlee M. P., i954 b. The effects of age upon calcium utilization and maintenance requirements in the bovine. J. Anim. Sci., 13, 25-36.

HANSARD S. L., CRowder H. M., 1957. The physiological behaviour of calcium in the Rat. J. Nitrition, 62, 325-339.

Lofgreen G. P., KLeiber M., I954. liurther studies on the availability of phosphorus in alfalfa hay. J. Anim. Sci., 13, 258-264.

Mathieu L. G., Suitri S. 1., ig6r. Phosphorus requirement of growing rabbits. J. Anim. Sci., 20, $5 \mathrm{IO}-5 \mathrm{I} 3$.

STERNKOPF G., 1963. Über Calcium-Phosphor-Bilanzen an Kaninchen bei liüterung mit Luzerne und Rotklee. Arch. fïr Tierernährung, 13, $247-253$.

Suzuki S., I962. Studies of calcium and phosphorus balance in Rabbits. I. The availability of various calcium salts. Res. Bull. Obihiro Zootech. Univ. Ser. I, 3, 330-336.

Suzukr S., I963a. Studies of calciun and phosphorus balance in Rabbits. II. Iiffects of the ratio of calcium to phosphorus in the ration on the metabolism of these elements. Res. Bull. Obihiro Zootech. Univ. Ser. I., 3, 398-4II.

SUzUKI S., I963 $b$. Studies of calcium and phosphorus balance in Rabbits. IV. Endogenous calcium and phosphorus. Res. Bull. Obihiro Zootech. Univ. Ser. I., 4, 63-73.

Thomas R. O., I.tovitz T. A., Rubin M. I., Geschickter C. F., i952. Iynamics of calcium metabolism : time distribution of intravenously administrated radiocalcium. A m. J. Physiol., 169, $568-575$.

WOOLEY J. G., Vickelsen O., I954. Effect of potassium, sodium or calcium on the growth of young rabbits fed purified diets containing different levels of fat and protein. J. Nutrition, 52, 59I-600. 